\title{
Quantum electronic transport in polarization-engineered GaN/InGaN/GaN tunnel junctions
}

Nicolas Cavassilas, ${ }^{1,}$ a) Yann Claveau, ${ }^{1}$ Marc Bescond, ${ }^{1}$ and Fabienne Michelini ${ }^{1}$

Aix Marseille Université, CNRS, Université de Toulon, IM2NP UMR 7334, 13397, Marseille, France

(Dated: 21 February 2017)

We theoretically investigate $\mathrm{GaN} / \mathrm{InGaN} / \mathrm{GaN}$ tunnel junctions grown along the wurtzite c-axis. We developed a dedicated quantum electronic transport model based on a 8-band k.p Hamiltonian coupled to the non-equilibrium Green's function formalism. We first show that the transmission is dominated by quantum states localized at the heterojunction. We also confirm,for thin InGaN layer, that current strongly increases with doping. On the other hand, for thick InGaN layers $(>8 \mathrm{~nm})$, our results show an unexpected low impact of doping on current. In this latter case, the spontaneous and the piezoelectric polarizations reduce the tunnel-barrier width to the InGaN layer thickness. We conclude that quantum electronic transport in such tunnel junctions is mainly controlled by interfaces with both polarizations and localized states.

a)Electronic mail: nicolas.cavassilas@im2np.fr 
Since the discovery of tunneling in degenerately doped Ge p-n junction reported by Esaki ${ }^{1}$, the tunnel junctions are employed in a broad range of electronic and optoelectronic devices such as light emitting diodes (LED),${ }^{2,3}$ laser diodes, ${ }^{4}$ multijunction solar cells, ${ }^{5}$ and tunnel field effect transistors ${ }^{6}$. Particularly, extensive research has led to tunnel junctions in III-As materials which have narrow bandgap and permit high doping. These two characteristics allow to obtain tunnel diodes exhibiting narrow depletion region and then a thin tunnel barrier. Since tunneling transmission depends exponentially on the width of the tunnel-barrier, such tunnel junctions exhibit large current density. On the contrary, in III-N materials, large bandgap and dopant solubility limitations in GaN leads to a wide depletion region that inhibits efficient interband tunneling. However, it has been shown that spontaneous and piezoelectric polarizations along the wurtzite c-axis improve tunneling in the so-called polarization-engineered GaN/III-N/GaN tunnel junctions. ${ }^{7}$ Such polarizations can reduce the space charge region (SCR) and therefore the width of tunnel-barrier. However, to date, the fabrication of an efficient tunnel junction using GaN-based materials is still challenging. ${ }^{3}$

Recently, in spite of these difficulties, GaN/InGaN/GaN tunnel junctions have been introduced in LEDs to replace the p-contact which limits the hole injection. ${ }^{2,3}$ Indeed, the low ionization efficiency of p-type dopants and low hole mobility lead to high parasitic resistances in III-N optoelectronics that cause significant losses. Such polarization-engineered tunnel junctions improve the efficiency and functionality of a variety of III-N emitters for visible and ultra violet. To optimize the design of these complicated junctions few theoretical calculations, based on the common Wentzel-Kramers-Brillouin approximation, have been conducted..$^{7,8}$ In this letter, we then propose a theoretical investigation beyond this approximation based on a sophisticated interband quantum transport model, to calculate the electrical current flowing through polarization-engineered GaN/InGaN/GaN tunnel junctions. ${ }^{6,9}$

In this work we use a 8-band k.p model in the wurtzite symmetry ${ }^{10}$, that explicitly accounts for all the interband couplings between the heavy-hole, the light-hole, the crystal-field split-off hole, and the first direct conduction bands. The strain induced effects in InGaN layer are also incorporated. Within this framework, we develop a one-dimensional (1D) description of the heterojunction along the c-axis, assuming the system invariant in the transverse plan. ${ }^{9}$ This $1 \mathrm{D}$ quantum electronic transport model, based on the non equilibrium Green's functions, is coupled to a 1D Poisson equation. This permits to self-consistently calculate the electrical potential along the junction including both piezoelectric and spontaneous charge polarizations at the GaN/InGaN interface following Ref. 11. All the considered parameters for GaN and 
InN are given in Ref. 10. To obtain parameters for InGaN we include a bowing parameter of $1.43 \mathrm{eV}^{10}$ for bandgap while for all other parameters we use a linear interpolation between the values for $\mathrm{GaN}$ and $\mathrm{InN}$.

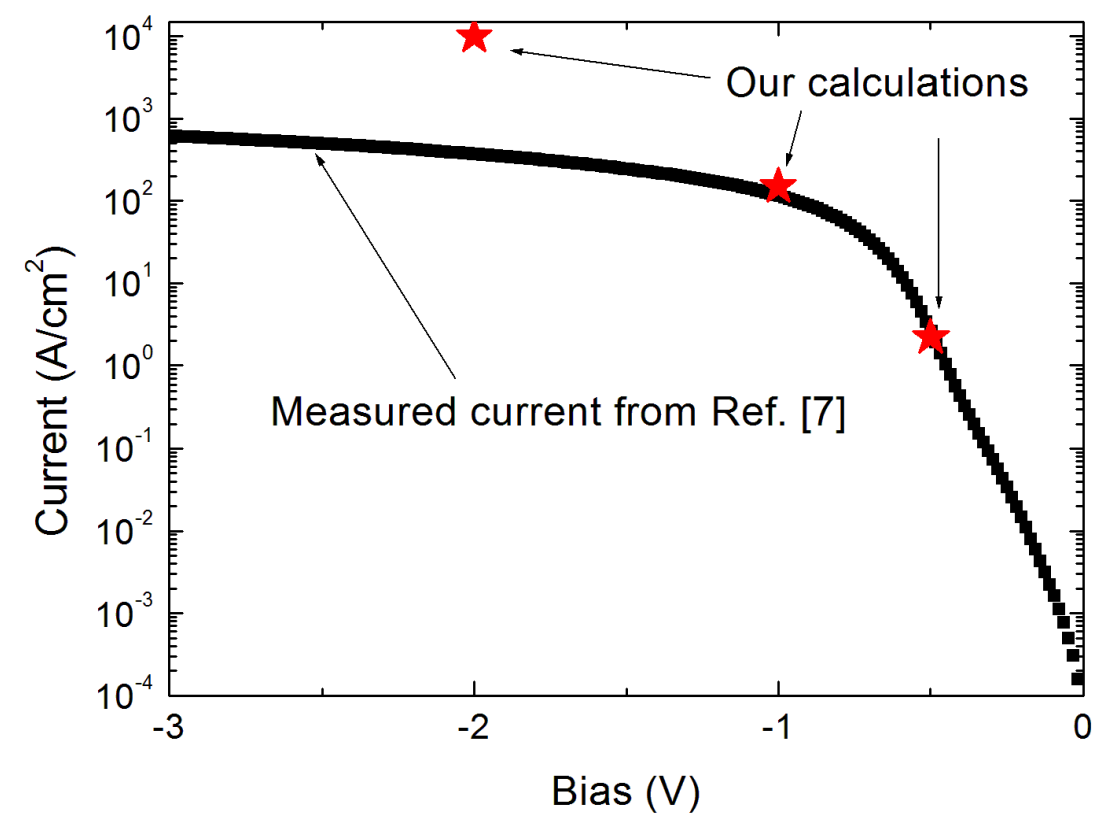

FIG. 1. Current characteristics of the diode (extracted from Ref. 7): $N_{D}=5 \cdot 10^{18} \mathrm{~cm}^{-3}, N_{A}=$ $10^{19} \mathrm{~cm}^{-3}$, the In content $x=0.33$ and the InGaN layer thickness $L=6.4 \mathrm{~nm}$. Crosses represent our calculations.

The model was first benchmarked on the experimental results of Krishnamoorthy and coauthors reported in Ref. 7 on the design and the demonstration of polarization-engineered $\mathrm{GaN} / \mathrm{InGaN} / \mathrm{GaN}$ tunnel junction diodes. The best current is obtained for a diode with $N_{D}=$ $5 \cdot 10^{18} \mathrm{~cm}^{-3}, N_{A}=10^{19} \mathrm{~cm}^{-3}$, the In content $x=0.33$ and the InGaN layer thickness $L=6.4$ nm. The corresponding current-bias characteristic obtained with our calculation at negative bias is shown in Fig. together with the curve extracted from the Fig. 3 of Ref. 7. We only investigated the reverse bias regime corresponding to the LED operation, i.e. injection of electrons from the valence band (VB) to the conduction band (CB). For low bias, without any fitting of parameters, our calculations are in excellent agreement since we obtain $2.2 \mathrm{~A} / \mathrm{cm}^{2}$ at $-0.5 \mathrm{~V}$ versus $2.6 \mathrm{~A} / \mathrm{cm}^{2}$ for the experimental value, and $150 \mathrm{~A} / \mathrm{cm}^{2}$ at $V=-1 \mathrm{~V}$ versus $118 \mathrm{~A} / \mathrm{cm}^{2}$. For higher bias, with $10^{4} \mathrm{~A} / \mathrm{cm}^{2}$ at $-2.0 \mathrm{~V}$ we clearly overestimate the measured current. Krishnamoorthy and co-authors attribute the increase of the differential resistance at bias exceeding $-1.0 \mathrm{~V}$ to series and contacts resistances and to self-heating which are not 
included in our model. Note that those effects related to the contacts do not have any impact once the tunnel junction is embedded in devices such as LED. This benchmark shows that, in reverse bias regime and for moderate bias, our model provides a reliable expectation value of the current. Moreover, as already discussed in GaAs, ${ }^{12}$ such a theoretical result shows that a large tunnel current density can be achieved even without assuming tunneling across impurities.

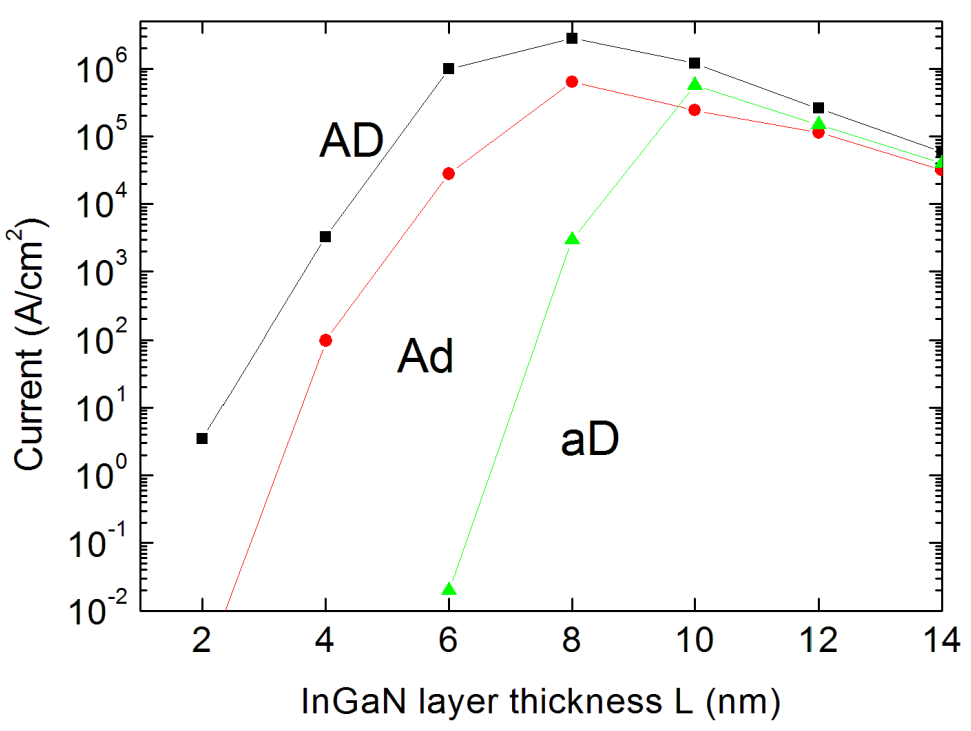

FIG. 2. Calculated current density at $V=-1 \mathrm{~V}$ in the tunnel junction $\mathrm{GaN} / \mathrm{In}_{0.33} \mathrm{Ga}_{0.67} \mathrm{~N} / \mathrm{GaN}$ versus the thickness of the InGaN layer for the three dopings AD $\left(N_{A}=10^{19} \mathrm{~cm}^{-3}, N_{D}=10^{20} \mathrm{~cm}^{-3}\right)$, Ad $\left(N_{A}=10^{19} \mathrm{~cm}^{-3}, N_{D}=10^{19} \mathrm{~cm}^{-3}\right)$ and $\mathbf{a D}\left(N_{A}=10^{18} \mathrm{~cm}^{-3}, N_{D}=10^{20} \mathrm{~cm}^{-3}\right)$.

Since the work published by Krishnamoorthy and co-authors, ${ }^{7}$ experimental limit of doping in GaN based devices, and particularly the n-doping, has increased. ${ }^{2,13}$ In the following we then assume $N_{A}=10^{19} \mathrm{~cm}^{-3}$ and $N_{D}=10^{20} \mathrm{~cm}^{-3}$ for the case denoted AD. In order to study the impact of the doping reduction we also consider two other cases: Ad with $N_{A}=10^{19} \mathrm{~cm}^{-3}$, $N_{D}=10^{19} \mathrm{~cm}^{-3}$ and aD with $N_{A}=10^{18} \mathrm{~cm}^{-3}, N_{D}=10^{20} \mathrm{~cm}^{-3}$ (capital D and A stand for high impurity concentrations). In all cases, the InGaN layer is undoped. Fig. 2 represents, for the three cases, the current versus the InGaN layer thickness $L$ calculated at $-1.0 \mathrm{~V}$ for an In content $x=0.33$. The case $\mathrm{AD}$ exhibits a maximum at $2.8 \times 10^{6} \mathrm{~A} / \mathrm{cm}^{2}$ for $L=8 \mathrm{~nm}$. For the two other cases, due to the reduced doping, the maxima are reduced but close: $6.4 \times 10^{5}$ $\mathrm{A} / \mathrm{cm}^{2}$ at $L=8 \mathrm{~nm}$ for Ad and $6.7 \times 10^{5} \mathrm{~A} / \mathrm{cm}^{2}$ at $L=10 \mathrm{~nm}$ for $\mathbf{a D}$.

For thin InGaN layer $(<8 \mathrm{~nm})$ the difference due to doping is very important. As shown in Fig. 3 where the local density of states and the band diagrams are represented for the three 


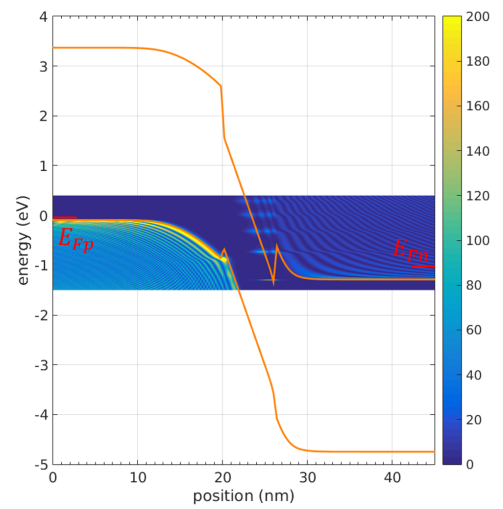

(a)

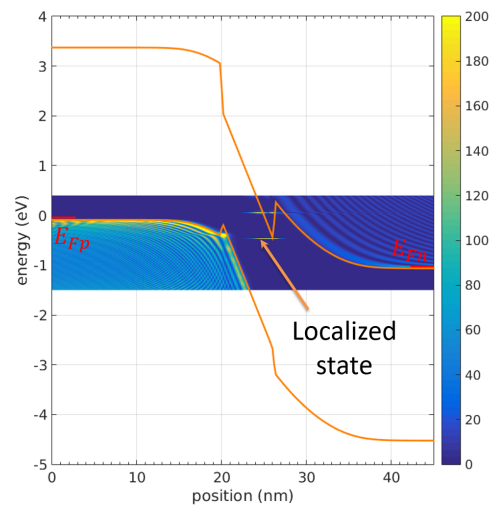

(b)

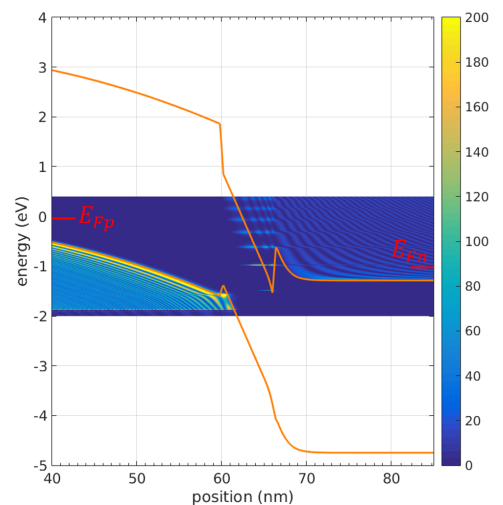

(c)

FIG. 3. Local density of states versus position (zoomed around the InGaN layer) and energy represented for the three tunnel junctions (a) $\mathbf{A D}\left(N_{A}=10^{19} \mathrm{~cm}^{-3}, N_{D}=10^{20} \mathrm{~cm}^{-3}\right)$, (b) Ad $\left(N_{A}=10^{19}\right.$ $\left.\mathrm{cm}^{-3}, N_{D}=10^{19} \mathrm{~cm}^{-3}\right)$ and (c) aD $\left(N_{A}=10^{18} \mathrm{~cm}^{-3}, N_{D}=10^{20} \mathrm{~cm}^{-3}\right)$. The In content is $x=0.33$ and the InGaN layer thickness is $6 \mathrm{~nm}$. We note the presence of localized states in the CB triangular quantum wells.

cases at $L=6 \mathrm{~nm}$, the SCR reduces when considering a large doping. This expected behavior is particularly critical for the p-doping that remains low. The consequence is a tunnel barrier which increases with a lower doping. Moreover, as shown in Fig. 3, some CB states localized in the triangular wells at the GaN/InGaN interfaces are visible. These states, due to the electronic reflection at the interface, cannot be observed without a full-quantum model. Yet, as shown in Fig. 4 where the transmission for Ad is represented, their contributions are essential. Indeed, the transmission profile is very peaked at the energy corresponding to the lower state in the $\mathrm{CB}$ triangular potential. It is then crucial to design junctions in order to have such localized states in the energy range of interest, i.e. between the two Fermi levels. Moreover, such a result shows that interfaces are determinant for the performance since the presence of localized states at the interfaces could greatly improve the current transmission. This is the reason why that the interfaces should be as abrupt as possible.

For thick InGaN layers $(>8 \mathrm{~nm}$ ), as shown Fig. 2, the current density for the three cases $\mathbf{A D}, \mathbf{a D}$ and $\mathbf{A d}$ are close. Indeed, as shown in Fig. 5 for $L=12 \mathrm{~nm}$, with a thick enough InGaN layer, the SCR is limited to such a layer, independently of the doping. This unexpected behavior is explained by the spontaneous and piezoelectric polarizations occurring in $\mathrm{GaN} / \mathrm{InGaN}$ heterojunctions along c-axis. Indeed, as shown Fig. 6 this behavior disappears when the polarization is artificially erased. In this case the SCR is simply determined by the 


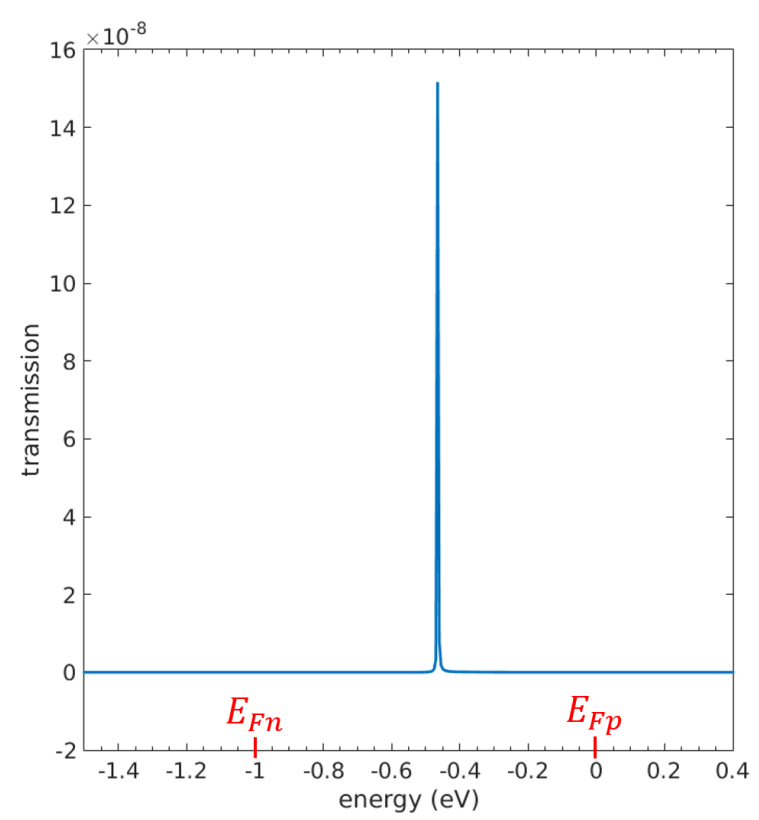

FIG. 4. Transmission with respect to the energy for the tunnel junction Ad with $L=6 \mathrm{~nm}$ and $x=0.33$. The peak corresponds to the energy of the lower localized state shown by the orange arrow in Fig. 3(a). Almost all the current flows through this localized state.

doping concentrations and is strongly thicker. This polarization induced behavior leads to the decrease of current when $L$ increases. It also explains that the current density is little depend on the doping for thick InGaN layer.

In conclusion, we have developed a model of electronic transport assuming a realistic multiband framework, the polarizations and the strain in III-N tunnel junctions. For moderate reverse bias this model is in excellent agreement with an experimental result. We have performed an investigation of the $\mathrm{GaN} / \mathrm{InGaN} / \mathrm{GaN}$ tunnel junctions with various doping concentrations and InGaN thicknesses. Due to polarizations for a large enough InGaN layer the SCR is controlled by the InGaN thickness. In this case, the differences due to doping are limited. On the other hand, for thinner InGaN layers the current strongly increases with doping. In all cases we show that states localized at the interfaces strongly enhance the tunneling. Finally this work shows that interfaces control current with both polarizations and localized states. Such behaviors could be reduced by non abrupt interfaces or a decrease of the In content. For example, we obtain a factor reduction of current maxima of about $10^{-3}$ for a In content going from 33 to 25 . 


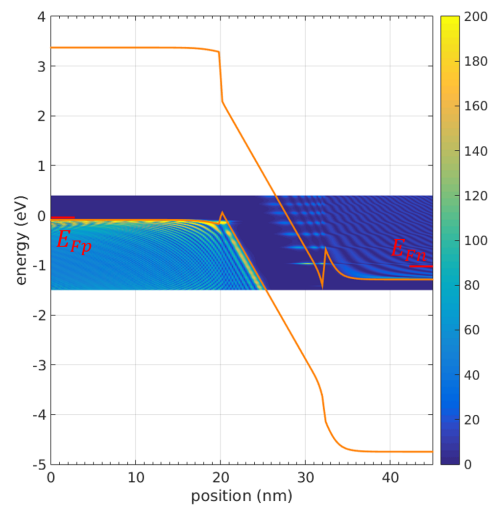

(a)

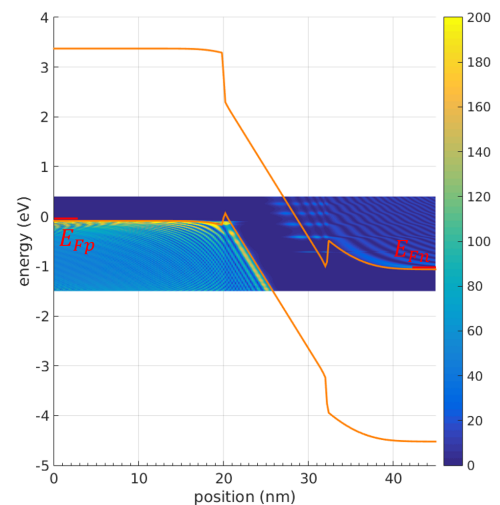

(b)

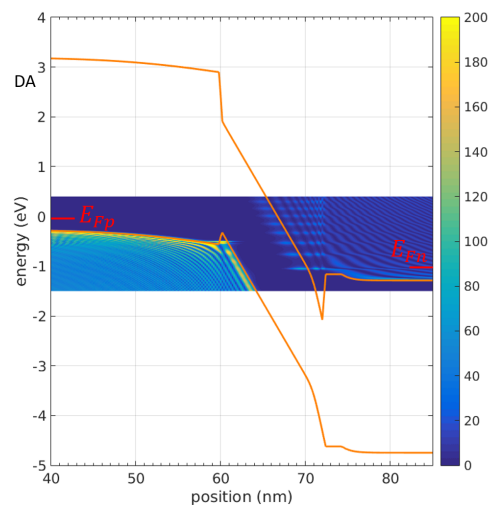

(c)

FIG. 5. Local density of states versus position (zoomed around the InGaN layer) and energy represented for the three tunnel junctions (a) $\mathbf{A D}\left(N_{A}=10^{19} \mathrm{~cm}^{-3}, N_{D}=10^{20} \mathrm{~cm}^{-3}\right)$, (b) Ad $\left(N_{A}=10^{19}\right.$ $\left.\mathrm{cm}^{-3}, N_{D}=10^{19} \mathrm{~cm}^{-3}\right)$ and (c) aD $\left(N_{A}=10^{18} \mathrm{~cm}^{-3}, N_{D}=10^{20} \mathrm{~cm}^{-3}\right)$. The In content is $x=0.33$ and the InGaN layer thickness is $12 \mathrm{~nm}$. The SCR approximatly equals the InGaN layer.

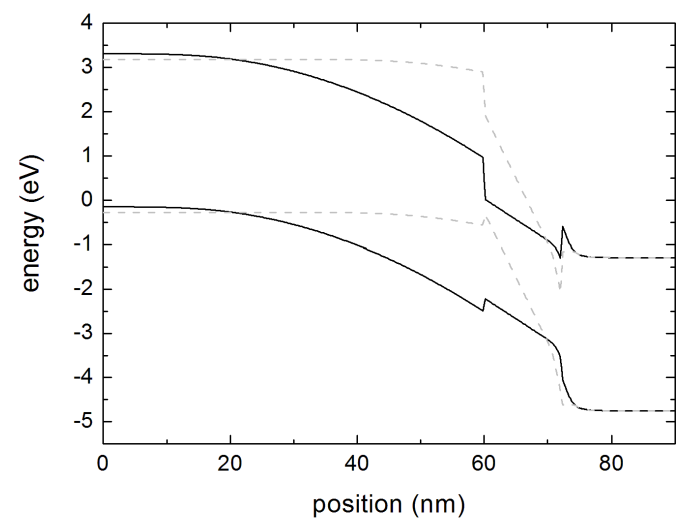

FIG. 6. Band diagrams of the cell aD with $L=12 \mathrm{~nm}$ without polarizations (solid line) and with polarizations (dash line). The polarizations limit the SCR at the InGaN layer while without polarizations such a SCR largely over gone in the p-type GaN which has a low doping.

\section{ACKNOWLEDGMENTS}

The financial support of the project ANR-14-CE26-0020-01 "Platofil" is acknowledged.

\section{REFERENCES}

${ }^{1}$ L. Esaki, Phys. Rev. 109, 603 (1958). 
${ }^{2}$ A. T. M. G. Sarwar, B. J. May, J. I. Deitz, T. J. Grassman, D. W. McComb, and R. C. Myers, Applied Physics Letters 107, 101103 (2015), http://dx.doi.org/10.1063/1.4930593.

${ }^{3}$ S. M. Sadaf, Y. H. Ra, T. Szkopek, and Z. Mi, Nano Letters 16, 1076 (2016), pMID: 26812264, http://dx.doi.org/10.1021/acs.nanolett.5b04215.

${ }^{4}$ B. P. Yonkee, E. C. Young, C. Lee, J. T. Leonard, S. P. DenBaars, J. S. Speck, and S. Nakamura, Opt. Express 24, 7816 (2016).

${ }^{5}$ J. P. Mailoa, C. D. Bailie, E. C. Johlin, E. T. Hoke, A. J. Akey, W. H. Nguyen, M. D. McGehee, and T. Buonassisi, Applied Physics Letters 106, 121105 (2015), http://dx.doi.org/10.1063/1.4914179.

${ }^{6}$ M. G. Pala, C. Grillet, J. Cao, D. Logoteta, A. Cresti, and D. Esseni, Journal of Computational Electronics 15, 1240 (2016).

${ }^{7}$ S. Krishnamoorthy, D. N. Nath, F. Akyol, P. S. Park, M. Esposto, and S. Rajan, Applied Physics Letters 97, 203502 (2010), http://dx.doi.org/10.1063/1.3517481.

${ }^{8}$ S. Krishnamoorthy, F. Akyol, P. S. Park, and S. Rajan, Applied Physics Letters 102, 113503 (2013), http://dx.doi.org/10.1063/1.4796041.

${ }^{9}$ N. Cavassilas, F. Michelini, and M. Bescond, Journal of Renewable and Sustainable Energy 6, 011203 (2014), http://dx.doi.org/10.1063/1.4828366.

${ }^{10}$ H. Saidi, S. Ridene, and H. Bouchriha, International Journal of Modern Physics B 29, 1550054 (2015), http://www.worldscientific.com/doi/pdf/10.1142/S021797921550054X.

${ }^{11}$ J. Piprek, Nitride Semiconductor Devices: Principles and Simulation (John Wiley and Son, 2007).

${ }^{12}$ K. Louarn, C. Fontaine, A. Arnoult, F. Olivié, G. Lacoste, F. Piquemal, A. Bounouh, and G. Almuneau, Semiconductor Science and Technology 31, 06LT01 (2016).

${ }^{13}$ H. Zhang, X. Dai, N. Guan, A. Messanvi, V. Neplokh, V. Piazza, M. Vallo, C. Bougerol, F. H. Julien, A. Babichev, N. Cavassilas, M. Bescond, F. Michelini, M. Foldyna, E. Gautier, C. Durand, J. Eymery, and M. Tchernycheva, ACS Applied Materials \& Interfaces 8, 26198 (2016), pMID: 27615556, http://dx.doi.org/10.1021/acsami.6b06414. 